\title{
An Optimal Transmission Strategy for IEEE 802.11 Wireless LANs: Stochastic Control Approach
}

\author{
Alexander W. Min and Kang G. Shin \\ Real-Time Computing Laboratory, Dept. of EECS \\ The University of Michigan, Ann Arbor, MI 48109-2121 \\ \{alexmin,kgshin\}@eecs.umich.edu
}

\begin{abstract}
The family of IEEE 802.11 Wireless Local Area Network (WLAN) standards supports multiple transmission rates in the physical layer (PHY). This multi-rate capability offers a viable means of coping with dynamically-fluctuating wireless channel conditions. We propose a cross-layer approach for the optimal PHY mode control to maximize the system goodput. Our key idea is to exploit the underlying channel fading characteristics and the history of PHY mode control and observations to infer the current channel condition, so that the optimal PHY mode may be selected. Assuming Rayleigh fading, we describe the receiver-side signal-to-noise ratio (SNR) fluctuations as a finite-state Markov channel (FSMC) model. Since the channel condition (i.e., fading level) is not directly observable by the transmitter, we formulate the PHY rate adaptation problem as a partially-observable Markov decision process (POMDP) to find the optimal transmission policy. We use the belief state vector to represent the channel state probabilistically. The belief state is updated solely based on the channel state-transition matrix and acknowledgement (ACK) information. Our evaluation results show that the POMDP-based rate adaptation outperforms two most well-known rate adaptation schemes, Auto Rate Fallback (ARF) and Adaptive ARF (AARF), in terms of average goodput under various fading conditions, and achieves up to $92 \%$ of the ideal performance.
\end{abstract}

\section{INTRODUCTION}

The IEEE 802.11 WLAN [1] is one of the most popular and widely-deployed wireless systems. The current family of IEEE 802.11 standards provides multiple data-transmission rates by adopting different modulation and channel-coding schemes at the physical layer (PHY). For example, the IEEE 802.11a supports eight $\mathrm{PHY}$ rates ranging from $6 \mathrm{Mbps}$ to $54 \mathrm{Mbps}$ in the $5 \mathrm{GHz}$ band; the IEEE $802.11 \mathrm{~b}$ supports four PHY rates from $1 \mathrm{Mbps}$ to $11 \mathrm{Mbps}$ in the $2.4 \mathrm{GHz}$ band; and the IEEE $802.11 \mathrm{~g}$ is an extension of IEEE $802.11 \mathrm{~b}$ and supports twelve PHY rates delivering up to $54 \mathrm{Mbps}$ in the $2.4 \mathrm{GHz}$ band. Currently, the IEEE 802.11 Working Group (WG) is driving the standardization of MIMO-based IEEE 802.11n, which is expected to support multiple PHY rates up to 600Mbps (raw rate) by introducing higher-order modulation schemes, such as 128-QAM or 256-QAM, combined with channel bonding.

In 802.11 WLAN environments, the signal-to-noise ratio (SNR) fluctuates due to various unpredictable phenomena including multi-path fading, path loss, shadowing, interferences, noise, etc. These channel dynamics are generally perceived as harmful to the network performance due to their unpredictability. However, the multi-rate capability of the IEEE 802.11 PHY provides a viable means of coping with timevarying wireless channel conditions. The basic idea of using the multi-rate capability is to make a trade-off between data rate and error probability, the higher the data rate, the higher the error probability. Therefore, it is natural to dynamically change the modulation scheme (i.e., PHY modes) according to time-varying channel conditions. This adaptive PHY mode selection mechanism is called rate adaptation.

The fundamental design goal of a rate adaptation scheme is to devise a PHY mode control mechanism that automatically selects the best PHY mode under a given channel condition. In general, however, the channel condition is not known a priori to the transmitter, thus needing an efficient way of estimating the channel condition. For this, various approaches have been employed by existing rate adaptation schemes. For example, RBAR [2] and OAR [3] are the PHY-layer approaches that control the PHY mode via direct channel SNR measurement at the receiver using RTS/CTS (Request-To-Send/Clear-To-Send) control messages. On the other hand, ARF [4] and AARF [5] are MAC-layer heuristics adapting the PHY mode by counting consecutive transmission successes/failures. Finally, SampleRate [6] and RRAA [7] use statistics over long- and short-term sampling intervals to maximize the average throughput.

None of the above rate-adaptation schemes, however, accurately estimates the channel condition due to the inherent limitations in their protocol design [5], [7]. For example, the SNR-measurement-based schemes (i.e., RBAR and OAR) suffer from the inaccuracy in channel condition estimation [6][8]. Heuristic approaches (i.e., ARF and AARF) cannot be optimized due to the limited adaptability of the counting method [7]. Finally, statistics-based approaches (i.e., SampleRate and RRAA) do not react quickly to dynamically-changing channel conditions due to their dependence on the sampling interval [7]. The non-optimality of existing schemes, in fact, stems from the lack of understanding of the physical-layer aspects of a wireless channel. For example, the time-correlated nature (i.e., memory) of a wireless channel fading process [9], which clearly benefits the channel estimation, is not considered in any of previous studies.

To overcome the above limitations of existing rateadaptation schemes, we propose a novel cross-layer transmission-control scheme in IEEE 802.11 WLANs that exploits the underlying channel fading process to derive an optimal transmission-control (i.e., PHY mode adaptation) policy. We assume a Rayleigh fading channel and model time-varying channel conditions as a finite-state Markov channel (FSMC). While the channel condition is not directly observable by the transmitter, the PHY mode use for packet transmission and the corresponding acknowledgement (ACK) packet can provide hints to estimate the current channel condition. Thus, we formulate the rate-adaptation problem as a partially-observable Markov decision process (POMDP). In POMDP, the condition of a wireless channel is estimated 
through a belief state vector, each element of which represents the likelihood of the channel being in a certain state at a given time. The belief state is updated at each packet transmission attempt by exploiting previous PHY mode control and corresponding observations (i.e., ACK) history. Based on the belief state, the transmitter selects the best PHY mode to maximize the system goodput.

The main contributions of this paper are three-fold. First, we provide an analytical framework for cross-layer design of optimal transmission control for IEEE 802.11 WLANs. Second, our scheme does not require direct SNR measurements in estimating the channel condition, which is known to be inaccurate and also incurs overhead (e.g., RTS/CTS) [6]-[8]. Third, our scheme accurately predicts the channel condition (even when there is no packet to transmit) instead of reacting to it after observing consecutive transmission failures or successes.

The rest of this paper is organized as follows. Section II presents the wireless channel model used in our scheme. Section III introduces the IEEE 802.11a MAC protocol and analyzes the probability of successful frame transmission for a given channel condition and PHY mode. Section IV describes the POMDP formulation for the transmission-control problem and also introduces the heuristic methods that we use. Section V presents our simulation results. Section VI makes conclusions and mentions future directions.

\section{Wireless ChANNEL MODEL}

To incorporate the physical channel characteristics in the design of a rate adaptation scheme, we need a channel model that describes the underlying physical-layer channel behaviors. The first-order Markov model has been recognized as a simple and accurate means of modeling Rayleigh fading channels [10][12]. While the original Markov model proposed in [10] is described in symbol-level time periods, it can be applied to packet-level IEEE 802.11 WLAN channels in a slow fading environment.

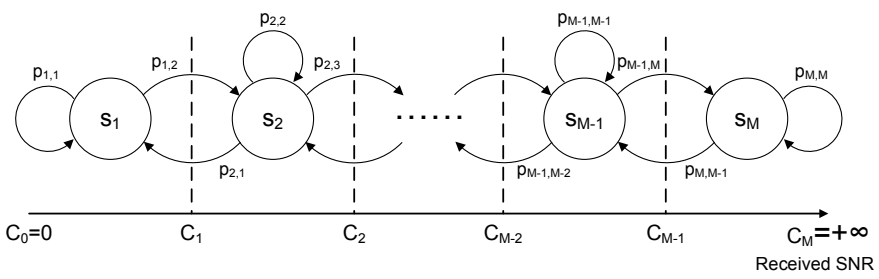

Fig. 1. Finite-state Markov channel (FSMC) model for a Rayleigh fading channel in IEEE 802.11 WLANs

Therefore, we model a Rayleigh fading channel as a finite-state Markov channel (FSMC) with state space $\mathcal{S}=$ $\left\{s_{1}, s_{2}, \ldots, s_{M}\right\}$ as shown in Fig. 1. The number of FSMC states (i.e., $M$ ) is a design parameter. In this paper, we adapt the method used in [10] to model a Rayleigh-fading channel as a FSMC. The FSMC is constructed by partitioning the range of the received SNR into a finite number of non-overlapping intervals. It is assumed that state transitions can occur only between adjacent states. This is a reasonable assumption for a slow-fading channel because channel state transitions are continuous and not abrupt.
We define the $i^{\text {th }}$ fading region as $\mathcal{D}_{i}=\left\{\gamma: C_{i-1} \leq \gamma<C_{i}\right\}$ where $0=C_{0}<C_{1}<\ldots<C_{M-1}<C_{M}=\infty$ represent the thresholds of the received SNR $\gamma$. Then, the channel is said to be in state $i, i \in\{1, \ldots, M\}$, if the received SNR $\gamma$ is in the fading region $\mathcal{D}_{i}$. Note that we set the SNR thresholds $C_{i}$ so that the steady-state probabilities are equiprobable (i.e., $\pi_{1}=\pi_{2}=\cdots=\pi_{M}$ ).

The expected downward transition rate $N_{C_{i}}$ of received SNR $\gamma$ across the threshold $C_{i}$ can be expressed as:

$$
N_{C_{i}}=\sqrt{\frac{2 \pi C_{i}}{\bar{\gamma}}} f_{D} \exp \left\{-\frac{C_{i}}{\bar{\gamma}}\right\},
$$

where $f_{D}$ is the maximum Doppler shift and $\bar{\gamma}$ is the average SNR.

Let $r_{B}$ denotes the block rate (per second) of the system. ${ }^{1}$ Then, the average block rate per second in state $i$ can be written as:

$$
R_{i}=\pi_{i} \times r_{B} \quad \forall i,
$$

where $\pi_{i}$ is the steady-state probability, which can be evaluated using the Rayleigh distribution as follows:

$$
\begin{aligned}
\pi_{i} & =\mathrm{P}\left(\gamma \in \mathcal{D}_{i}\right)=\int_{C_{i-1}}^{C_{i}} \frac{1}{\bar{\gamma}} e^{-\frac{x}{\bar{\gamma}}} \mathrm{d} x \\
& =\exp \left\{-\frac{C_{i-1}}{\bar{\gamma}}\right\}-\exp \left\{-\frac{C_{i}}{\bar{\gamma}}\right\} .
\end{aligned}
$$

Then, based on Eqs. (1), (2), and (3), the state-transition probabilities can be approximated by the expected ratio of the number of level-crossings at $C_{i}$ to the average number of blocks per second in state $i$. That is,

$$
p_{i, i+1} \approx \frac{N_{C_{i}}}{R_{i}} \quad i=1, \ldots, M-1
$$

and

$$
p_{i, i-1} \approx \frac{N_{C_{i-1}}}{R_{i}} \quad i=2, \ldots, M .
$$

The values of $p_{1,1}, p_{M, M}$, and $p_{i, i}$ are given as:

$$
p_{1,1}=1-p_{1,2}, \quad p_{M, M}=1-p_{M, M-1}
$$

and

$$
p_{i, i}=1-p_{i, i+1}-p_{i, i-1} \quad i=2, \ldots, M-1 .
$$

The thus-obtained probabilities can be used by the transmitter to estimate the channel state. This will be detailed in Section IV.

Note that our FSMC model can be easily extended for the general Nakagami- $m$ model which encompasses a large class of fading channels (e.g., Rayleigh and Rician) [13]. Moreover, the FSMC model can be used to model the SNR fluctuations in 802.11 WLAN channels via SNR measurements even without the Rayleigh or Rician fading assumption [14]-[16].

\section{IEEE 802.11A WLANS}

In this section, we briefly describe the multi-rate capability and frame formats of IEEE 802.11a PHY. We then derive the average successful transmission probability for each combination of PHY mode and channel state of the FSMC model.

\footnotetext{
${ }^{1}$ In this paper, a single block corresponds to the average time interval of back-to-back frame transmission.
} 


\section{A. IEEE 802.11a Physical Layer}

The IEEE 802.11a PHY offers eight PHY modes: BPSK, QPSK, 16-QAM, and 64-QAM, combined with various rate convolutional coding for forward error correction (FEC), as shown in Table I. We denote by $\mathcal{M}$ a set of the eight PHY modes of the IEEE 802.11a throughout the paper.

TABLE I

EIGHT PHY MODES OF THE IEEE 802.11A

\begin{tabular}{|c|c|c|c|c|}
\hline Mode & Modulation & Coding Rate & Data Rate & BpS \\
\hline \hline 1 & BPSK & $1 / 2$ & $6 \mathrm{Mbps}$ & 3 \\
\hline 2 & BPSK & $3 / 4$ & $9 \mathrm{Mbps}$ & 4.5 \\
\hline 3 & QPSK & $1 / 2$ & $12 \mathrm{Mbps}$ & 6 \\
\hline 4 & QPSK & $3 / 4$ & $18 \mathrm{Mbps}$ & 9 \\
\hline 5 & $16-\mathrm{QAM}$ & $1 / 2$ & $24 \mathrm{Mbps}$ & 12 \\
\hline 6 & $16-\mathrm{QAM}$ & $3 / 4$ & $36 \mathrm{Mbps}$ & 18 \\
\hline 7 & $64-\mathrm{QAM}$ & $2 / 3$ & $48 \mathrm{Mbps}$ & 24 \\
\hline 8 & $64-\mathrm{QAM}$ & $3 / 4$ & $54 \mathrm{Mbps}$ & 27 \\
\hline
\end{tabular}

The format of the IEEE 802.11a data frame is shown in Fig. 2. In the MAC layer, the higher layer data (i.e., frame body) is encapsulated by adding the MAC header and frame check sequence (FCS). This is called the MAC Protocol Data Unit (MPDU). Then the MPDU is passed down to the PHY layer where the PLCP preamble and PLCP header are added to construct the PLCP Protocol Data Unit (PPDU) when the frame is transmitted to the wireless medium. Note that, the PLCP preamble and PLCP header except the SERVICE field are always transmitted at the lowest PHY rate (i.e., 6Mbps), while any PHY mode can be used for the following part including the MPDU. The format of the ACK frame is shown in Fig. 3. Note that these frame format information will be used in approximating the successful frame transmission probabilities in the next subsection.

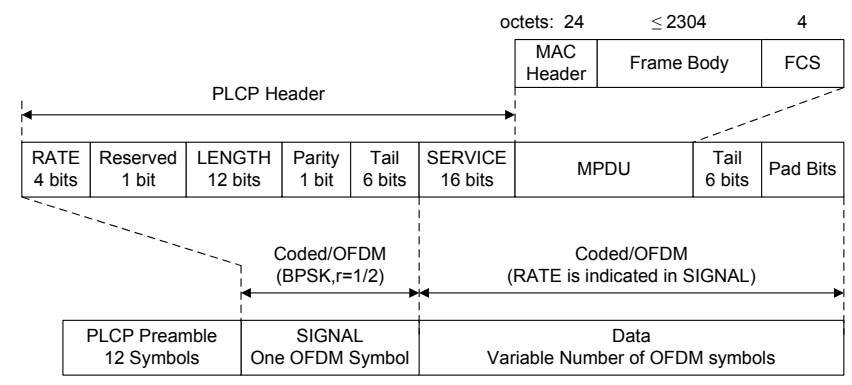

Fig. 2. PPDU frame format of the IEEE 802.11a PHY

\begin{tabular}{|l|c|c|c|}
\multicolumn{1}{c}{ octets: 2} & 2 & 6 & 4 \\
\begin{tabular}{|c|c|} 
Frame \\
Control
\end{tabular} & Duration & $\begin{array}{l}\text { Receiver } \\
\text { Address }\end{array}$ & FCS \\
\hline
\end{tabular}

Fig. 3. ACK frame format of the IEEE 802.11a PHY

\section{B. BER Upper Bounds for IEEE 802.11a PHY modes}

Here we derive the bit-error-rate (BER) upper bounds of the IEEE 802.11a PHY modes. For channel coding scheme, we assume a binary convolutional code with hard-decision Viterbi

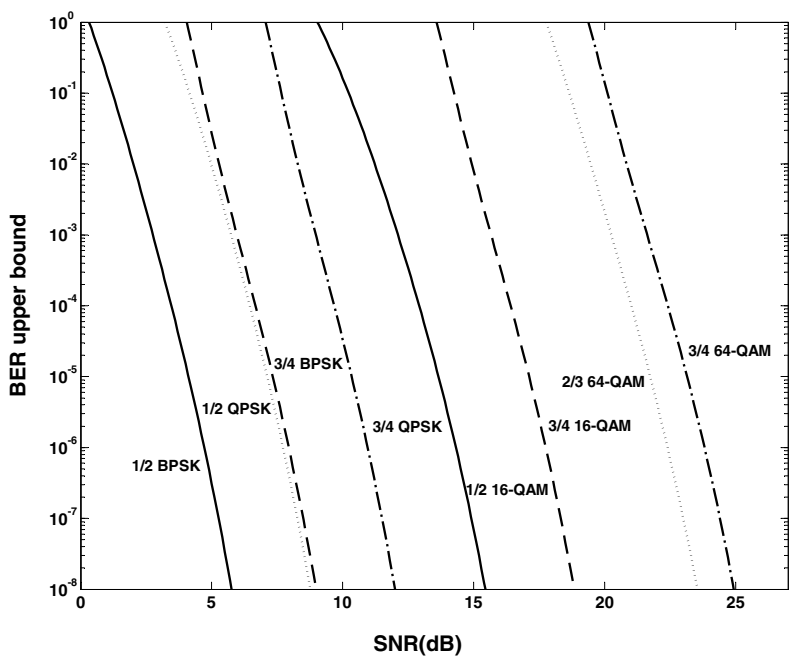

Fig. 4. BER upper bounds of the IEEE 802.11a PHY modes

decoding. Then, given the average symbol SNR $\gamma$, the symbol error rates of BPSK and M-QAM modulation can be calculated as follows [9]: ${ }^{2}$

$$
p_{s}^{m}(\gamma)=Q(\sqrt{2 \gamma})
$$

and

$$
p_{s}^{m}(\gamma)=1-\left(1-\frac{2(\sqrt{M}-1)}{\sqrt{M}} Q\left(\sqrt{\frac{3 \gamma}{M-1}}\right)\right)^{2},
$$

where $m \in \mathcal{M}$ is the adopted PHY mode.

By applying the union bound, an upper bound of the bit error probability $p_{b}^{m}$ can be computed as [9]:

$$
p_{b}^{m}(\gamma) \leq \sum_{d=d_{\text {free }}}^{\infty} a_{d} f(d) P_{2}^{m}(d, \gamma),
$$

where $d_{\text {free }}$ is the free distance of the convolutional code, $a_{d}$ is the total number of error events of weight $d$, and $f(d)$ is the number of bit errors associated with a path of distance $d$ from the all-zero path. $P_{2}(d, \gamma)$ is called the pairwise error probability which is the probability of an incorrect sequence of distance $d$ away from the correct sequence being chosen by the Viterbi decoder. Instead of direct calculation of $P_{2}^{m}(d, \gamma)$, we can use the Chernoff upper bound to obtain [9]:

$$
P_{2}^{m}(d, \gamma)<\left[4 p^{m}(\gamma)\left(1-p^{m}(\gamma)\right)\right]^{d / 2} .
$$

Then, by substituting Eq. (9) into Eq. (8), the upper bound on bit error probability (BER) can be expressed as follows [9]:

$$
p_{b}^{m}(\gamma)<p_{u b}^{m}(\gamma)=\left.\frac{d T(D, N)}{d N}\right|_{N=1, D=\sqrt{4 p(\gamma)(1-p(\gamma))}} .
$$

Therefore, the BER upper bound can be computed by using the transfer function $T(D, N)$ or the values $a_{d}$ and $f(d)$. Fig. 4 shows the BER upper bounds of the IEEE 802.11a PHY modes. Based on the BER upper bound for each modulation scheme, the probability of successful data frame transmission can be approximated as discussed in the following subsection.

\footnotetext{
${ }^{2}$ The Q-function is defined as $Q(x)=\int_{x}^{\infty} \frac{1}{\sqrt{2 \pi}} e^{-y^{2} / 2} \mathrm{~d} y$.
} 


\section{Probability of Successful Transmission}

We consider a data frame transmission as successful if the frame is decoded at the receiver without an error and the corresponding acknowledgement (ACK) packet is correctly received at the transmitter. Therefore, the successful frame transmission probability, denoted by $p_{s u c c}^{m}(\gamma)$, can be approximated as:

$$
p_{\text {succ }}^{m}(\gamma) \approx\left(1-p_{c o l}\right)\left(1-p_{\text {data }}^{m}(\gamma)\right)\left(1-p_{a c k}(\gamma)\right)
$$

where $p_{c o l}$ is the collision probability, and $p_{\text {data }}^{m}(\gamma)$ and $p_{a c k}(\gamma)$ are the data frame and the acknowledgement (ACK) frame transmission error probabilities given SNR $\gamma$ and PHY mode $m$, respectively.

Let $L$ denote the data payload length (in bytes). Then the data frame error probability can be computed as follows:

$$
p_{\text {data }}^{m}(\gamma)=1-\left(1-p_{u b}^{1}(\gamma)\right)^{24}\left(1-p_{u b}^{m}(\gamma)\right)^{(28+L) \cdot 8+22} \text {. }
$$

For the sake of simplicity, we assume that the ACK packet is always transmitted at the lowest PHY rate (i.e., 6Mbps). ${ }^{3}$ Then, the $p_{a c k}(\gamma)$ can be computed as follows:

$$
p_{a c k}(\gamma)=1-\left(1-p_{u b}^{1}(\gamma)\right)^{14 \cdot 8+46} \text {. }
$$

Since the optimal PHY mode would be determined based on the discrete set of channel states, an average successful frame transmission probability on each channel state should be calculated. Assuming the channel is in state $i$ and PHY mode $m$ is used, the average successful transmission probability can be found by integrating the $p_{s u c c}^{m}(\gamma)$ given by Eq. (11) over the Rayleigh fading distribution $f_{R}(\gamma)$ in the $i^{\text {th }}$ fading region $\mathcal{D}_{i}$. Then, it can be further approximated by using the average SNR $\bar{\gamma}_{i}$. That is,

$$
\begin{aligned}
p_{\text {succ }, i}^{m} & =\frac{\int_{C_{i-1}}^{C_{i}} p_{\text {succ }}^{m}(\gamma) f_{R}(\gamma) \mathrm{d} \gamma}{\int_{C_{i-1}}^{C_{i}} f_{R}(\gamma) \mathrm{d} \gamma} \\
& \approx p_{\text {succ }}^{m}\left(\bar{\gamma}_{i}\right) \quad \forall i
\end{aligned}
$$

where

$$
\bar{\gamma}_{i}=\frac{\int_{C_{i-1}}^{C_{i}} \gamma f_{R}(\gamma) \mathrm{d} \gamma}{\int_{C_{i-1}}^{C_{i}} f_{R}(\gamma) \mathrm{d} \gamma}=\frac{C_{i} e^{\frac{C_{i}}{\bar{\gamma}}}-C_{i-1} e^{\frac{C_{i-1}}{\bar{\gamma}}}}{e^{\frac{C_{i}}{\bar{\gamma}}}-e^{\frac{C_{i-1}}{\bar{\gamma}}}}-\bar{\gamma} .
$$

Note that since the average successful transmission probability in Eq. (14) is approximated from the BER upper bounds, it may significantly different from the empirical values, especially for large data frames. In practice, an empirical BERSNR curve provided by chip manufacturers (e.g., [17]) can be used to have more accurate PHY mode control.

\section{DECISION-THEORETIC FRAMEWORK FOR TRANSMISSION CONTROL}

In this section, we develop a POMDP-based decisiontheoretic approach for PHY mode adaptation for IEEE 802.11a WLANs. Since it is computationally too expensive to solve POMDP directly, we introduce heuristics to find a near-optimal PHY mode adaptation policy with reduced computational overhead.

\footnotetext{
${ }^{3}$ According to the IEEE 802.11a MAC specification, the ACK frame is transmitted at the highest PHY rate among the basic service set (BSS) basic rate that is less than or equal to the rate of the data frame. BSS basic rate set of IEEE $802.11 \mathrm{a}$ is $\{6 \mathrm{Mbps}, 12 \mathrm{Mbps}, 18 \mathrm{Mbps}\}$.
}

\section{A. POMDP Formulation}

Since the true channel state is not completely observable by the transmitter, the PHY mode control problem is inherently a POMDP problem as depicted in Fig. 5. The main advantages of using POMDP for rate adaptation is two-fold. First, the belief state vector, which represents the estimated channel state, enables more sophisticated PHY mode control than existing probing-based rate adaptation schemes (e.g., ARF, AARF, and SampleRate). Second, various performance metrics, such as goodput, error rate, delay, and power consumption, can be incorporated in the PHY mode control via the reward function.

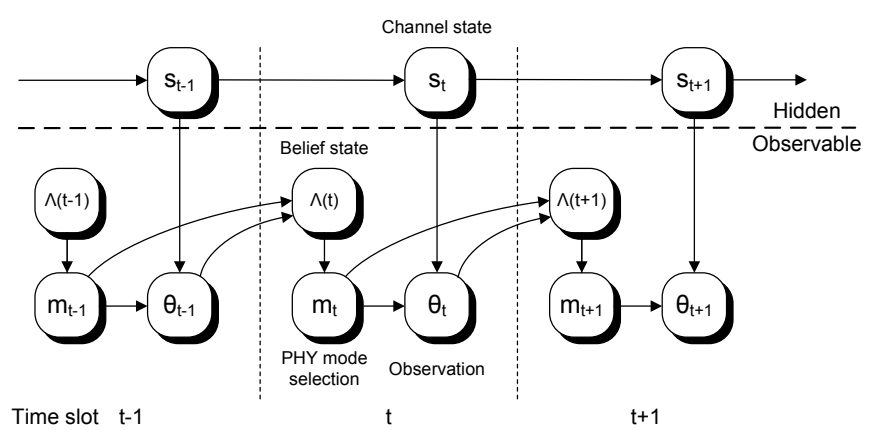

Fig. 5. A graphical model of the POMDP for PHY mode adaptation

POMDP is described formally as: (i) a set of time slots $\mathcal{T}=\{1,2, \ldots, t\}$ over which PHY mode decisions to be made; (ii) a set of states $\mathcal{S}$; (iii) a set of controls $\mathcal{U}$; (iv) a set of state-transition probabilities $\mathcal{P}$; (v) a set of observations $\Theta$; (vi) a set of observation probabilities $\Omega$; and (vii) a set of rewards $\mathcal{R}$. We assume a discrete-time POMDP model since PHY mode selections are made for each frame transmission. However, a frame transmission interval is not fixed in the 802.11a MAC protocol (e.g., random backoff). Thus, we consider an average frame transmission time, which is denoted as $\mathrm{E}\left[t T x_{\text {_D Duration }}\right]$, as a duration of each time slot in $\mathcal{T}$. The derivation of the average frame transmission time will be shown later in this section. The $\mathcal{S}$ and $\mathcal{P}$ corresponds to the set of FSMC model states and its state-transition probability matrix, respectively, as defined in Section II. The set of controls $\mathcal{U}$ consists of the eight PHY modes in the IEEE 802.11a PHY (i.e., $\mathcal{U} \triangleq \mathcal{M}$ ). At each time slot $t$, the true channel state $s_{t} \in \mathcal{S}$ is hidden from the transmitter, while an observation $\theta_{t} \in \Theta=\{0$ (failure), 1 (success) $\}$ can be seen. The observation $\theta_{t}$ is equal to 1 if the ACK frame is correctly received, and 0 otherwise. The stochastic process $\left\{\theta_{t}: t \in \mathbb{Z}_{+}\right\}$is called the observation process and the observation probability $\omega\left(s_{t}, m_{t}, \theta_{t}\right) \in \Omega$ can be expressed as:

$$
\omega\left(s_{t}, m_{t}, \theta_{t}\right)=\mathrm{P}\left(\theta_{t} \mid s_{t}, m_{t}\right) \quad s_{t} \in \mathcal{S}, \theta_{t} \in \Theta, m_{t} \in \mathcal{M},
$$

which is the conditional probability of observation $\theta_{t}$ given channel state $s_{t}$ and PHY mode $m_{t}$. Therefore, it can be approximated as:

$$
\omega\left(s_{t}, m_{t}, \theta_{t}\right) \approx \begin{cases}p_{s u c c, s_{t}}^{m_{t}} & \text { if } \theta_{t}=1 \\ 1-p_{\text {succ } s_{t}}^{m_{t}} & \text { if } \theta_{t}=0,\end{cases}
$$

where $p_{\text {succ }, i}^{m}$ is the average transmission success probability derived in Section III. 


\section{B. Channel State Estimation}

If we let $\lambda_{i}(t) \triangleq \mathrm{P}\left(s_{t}=i \mid \Lambda(1), m_{1: t}, \theta_{1: t}\right)$, then the belief state vector $\Lambda(t) \triangleq\left[\lambda_{1}(t), \lambda_{2}(t), \ldots, \lambda_{M}(t)\right]$ represents the estimated probability distribution of channel states at time slot $t$. Each element $\lambda_{i}(t)$ denotes the conditional probability that the channel is in state $i$ at time slot $t$ prior to the state transition, given all the PHY mode decision and the corresponding observation (i.e., ACK) history. Note that $\lambda_{i}(t) \geq 0 \forall i \in \mathcal{M}$ and $\sum_{i=1}^{M} \lambda_{i}(t)=1 \forall t \in \mathcal{T}$ since the channel must reside in one of the $M$ states at any given time slot. Each element of the belief state vector can be updated recursively for every time slot using the Bayes rule:

$$
\begin{aligned}
\lambda_{j}(t+1) & \triangleq \Psi_{j}\left(\Lambda(t) \mid \theta_{t}, m_{t}\right) \\
& =\frac{\sum_{i=1}^{M} \lambda_{i}(t) p_{i j} \omega\left(j, m_{t}, \theta_{t}\right)}{\sum_{i=1}^{M} \sum_{j=1}^{M} \lambda_{i}(t) p_{i j} \omega\left(j, m_{t}, \theta_{t}\right)} \quad \forall j \in \mathcal{S},
\end{aligned}
$$

where $p_{i j}$ is the state-transition probability derived in Section II. We denote $\lambda_{i}(t+1) \triangleq \lambda_{i}\left(t+1 \mid \theta_{1: t}, m_{1: t}\right)$ for brevity. Clearly, the belief state vector $\Lambda(t)$ summarizes all the necessary information for making PHY mode decision at time slot $t$. Note that even if there is no observation (e.g., no packet to transmit or contention) in any time slot, the belief vector can be still updated based on the channel state-transition probabilities, thus allowing the transmitter to keep track of the actual channel conditions. The computational complexity in updating the belief state vector is $O\left(M^{2}\right)$. The sequence of operations in each time slot is depicted in Fig. 6.

\section{Definition of Reward}

In order to design the optimal transmission policy, we need to specify a criterion for optimality. We define a real-valued reward function $\mathcal{R}: \mathcal{S} \times \mathcal{M} \rightarrow \mathbb{R}$ which indicates the reward earned in a time slot when the process is in state $i \in \mathcal{S}$ and PHY mode control $m \in \mathcal{M}$ is applied in that time slot. For our PHY mode control problem, we use the expected average reward per unit time over an infinite horizon (i.e., long-term average goodput) as the optimality criterion.

Since our objective is to maximize system goodput, we want to define the reward function so as to accurately represent the expected goodput corresponding to the adopted PHY mode and belief state at each time slot. Therefore, the expectation of reward function should be represented as:

$$
\mathrm{E}[\mathcal{R}(i, m)] \triangleq \frac{\text { avg. \# bits transmitted }}{\text { avg. transmission time duration }} .
$$

First, the number of data bits transmitted in a time slot can be written as:

$$
n T x_{-} \text {Bits }= \begin{cases}L \times 8 & \text { w.p. } \quad p_{\text {succ }, i}^{m} \\ 0 & \text { w.p. } 1-p_{\text {succ }, i}^{m},\end{cases}
$$

where $L$ is data payload length in bytes. Recall that $p_{s u c c, i}^{m}$ is the probability of successful frame transmission associated with the channel state $i$ and PHY mode $m$.

For each successful frame transmission, the transmitter sends a data frame, and then the receiver responds with an ACK frame after waiting for SIFS. Once the transmitter receives the ACK frame correctly, after waiting for DIFS, it

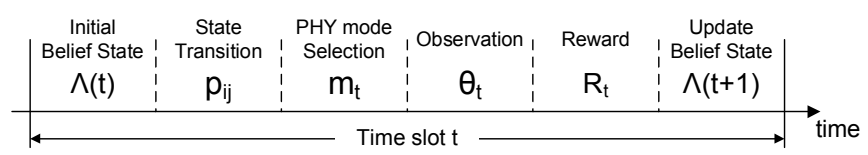

Fig. 6. The sequence of operations of POMDP in a time slot

TABLE II

IEEE 802.11A PHY PARAMETERS

\begin{tabular}{|l|l|l|}
\hline Parameter & Value & Comments \\
\hline \hline$t$ SlotTime & $9 \mu \mathrm{s}$ & Slot time \\
\hline$t$ SIFSTime & $16 \mu \mathrm{s}$ & SIFS time \\
\hline$t$ DIFSTime & $34 \mu \mathrm{s}$ & DIFS = SIFS $+2 \times$ Slot \\
\hline$a C W m i n$ & 15 & min contention window size \\
\hline$a C W m a x$ & 1023 & max contention window size \\
\hline$t$ PLCPPreamble & $16 \mu \mathrm{s}$ & PLCP preamble duration \\
\hline$t$ PLCP_SIG & $4 \mu \mathrm{s}$ & PLCP SIGNAL field duration \\
\hline$t$ Symbol & $4 \mu \mathrm{s}$ & OFDM symbol interval \\
\hline
\end{tabular}

performs a random backoff before transmitting the next frame. However, if the data frame transmission fails, the transmitter waits for an ACK timeout period, then waits for DIFS, and then performs a random backoff. Therefore, the total transmission time for a single data frame can be expressed as:

$$
\begin{aligned}
& \text { tTx_Duration } \\
& = \begin{cases}T_{\text {data }}^{m}+\text { tSIFSTime }+ \text { tDIFSTime } & \\
\bar{T}_{\text {back }}(0)+T_{\text {ack }} & \text { w.p. } p_{\text {succ }, i}^{m} \\
T_{\text {data }}^{m}+t \text { tSIFSTime }+t \text { SlotTime } & \\
\text { tDIFSTime }+\bar{T}_{\text {back }, i}^{m}+T_{\text {ack }} & \text { w.p. } 1-p_{\text {succ }, i}^{m},\end{cases}
\end{aligned}
$$

where the PHY layer parameters of the IEEE 802.11a are listed in Table II. $T_{\text {data }}^{m}$ and $T_{a c k}$ represent the time durations required for transmitting the data and ACK frames, respectively. They can be computed based on Figs. 2 and 3 as follows [18]:

$$
\begin{aligned}
T_{\text {data }}^{m}= & \text { tPLCPPreamble }+ \text { tPLCP_SIG } \\
& +\left\lceil\frac{28+(16+6) / 8+L}{B p S(m)}\right\rceil \cdot t \text { Symbol }
\end{aligned}
$$

and

$$
\begin{aligned}
T_{a c k}= & \text { tPLCPPreamble }+ \text { tPLCP_SIG } \\
& +\left\lceil\frac{14+(16+6) / 8}{B p S(1)}\right\rceil \cdot t \text { Symbol } \\
= & 40 \mu \mathrm{s},
\end{aligned}
$$

where $B p S(m)$ is the Bytes-per-Symbol for PHY mode $m$ in IEEE 802.11a (see Table I in Section III). $\bar{T}_{b a c k, i}^{m}$ in Eq. (20) represents the average random backoff interval of all re-transmission attempts in state $i$ with PHY mode $m$. Note that the average random backoff period for the $i^{\text {th }}$ retransmission attempt is given as follows [18]:

$$
\bar{T}_{b a c k}(i)=\frac{\min \left[2^{i} \cdot(a C W \min +1)-1, a C W \max \right]}{2} \times t \text { SlotTime. }
$$




$$
\begin{aligned}
& \mathrm{E}[\mathcal{R}(i, m)]=\frac{\mathrm{E}\left[n T x \_ \text {Bits }\right]}{\mathrm{E}[t \text { Tx_Duration }]} \\
& =\frac{L \times 8 \times p_{\text {succ }, i}^{m}}{p_{\text {succ }, i}^{m} \times\left(T_{\text {data }}^{m}+t \text { SIFSTime }+T_{a c k}+t \text { DIFSTime }+\bar{T}_{\text {back }}(0)\right)+\left(1-p_{\text {succ }, i}^{m}\right) \times\left(T_{\text {data }}^{m}+T_{a c k}+t \text { SIFSTime }+t \text { SlotTime }+t \text { DIFSTime }+\bar{T}_{\text {back }, i}^{m}\right)} \\
& =\frac{L \times 8 \times p_{\text {succ }, i}^{m}}{p_{\text {succ }, i}^{m} \times \bar{T}_{\text {back }}(0)+\left(1-p_{\text {succ }, i}^{m}\right) \times\left(t \text { SlotTime }+\bar{T}_{\text {back }, i}^{m}\right)+T_{\text {data }}^{m}+t \text { SIFSTime }+T_{\text {ack }}+t \text { tDIFSTime }} .
\end{aligned}
$$

Then, the average backoff period of all transmission attempts can be computed as:

$$
\bar{T}_{b a c k, i}^{m}=\sum_{k=0}^{\hat{n}}\left(1-p_{s u c c, i}^{m}\right)^{k} p_{s u c c, i}^{m} \times \bar{T}_{\text {back }}(k),
$$

where $\hat{n}$ is the maximum number of re-transmission attempts.

Finally, the expected reward which represents the effective goodput given channel state $i$ and PHY mode $m$ can be obtained as shown in Eq. (25).

\section{Optimal PHY Mode Control Policy}

Our objective is to maximize the goodput by employing an optimal policy $\pi^{*}=\left\{\mu_{1}, \mu_{2}, \cdots\right\}$, which specifies the optimal PHY mode decision rule for every time slot $t \in \mathcal{T}$ (i.e., every frame transmission). The policy is said to be stationary if it has a form $\pi=\{\mu, \mu, \cdots\}$. For brevity, we use $\mu$ to denote a stationary policy. Then the policy $\mu$ is said to be optimal if it satisfies:

$$
J_{\mu}(i)=J^{*}(i)=\max _{\pi} J_{\pi}(i) \quad \forall i \in \mathcal{S},
$$

where $J_{\pi}(i)$ denotes the long-term average reward associated with an initial state $i$ and a policy $\pi$.

Note that the policy is a mapping from belief state vector $\Lambda(t)$ to the PHY mode $m$ as:

$$
\mu: \Lambda(t) \in[0,1]^{M} \rightarrow m \in \mathcal{M} \quad \forall t \in \mathcal{T} .
$$

This indicates that, in our POMDP problem, we need to consider the entire space of belief states to find the optimal solution. In other words, we need to apply MDP for an uncountable infinite state space. For this reason, the solution of an infinite horizon stochastic POMDP is undecidable for general but bounded rewards [19]. On the other hand, for general MDP problems, an optimal policy can be found by assuming that states are completely observable.

Therefore, we first consider a Markov decision process (MDP) by assuming that the channel state is perfectly known for every time slot. Our objective is then to find an optimal stationary transmission policy of MDP which maximizes the expected long-term average reward. That is,

$$
\mu_{M D P}^{*}=\arg \max _{\pi} \lim _{t \rightarrow \infty} \frac{1}{t} \mathrm{E}_{\pi}\left[\sum_{t=1}^{\infty} \mathcal{R}(i, m) \mid \Lambda(1)\right],
$$

where $E_{\pi}$ represents the conditional expectation given that policy $\pi$ is employed and $\Lambda(1)$ is the initial belief state vector, which can be an arbitrary distribution.
The maximum average reward $\xi^{*}$ with some vector $h^{*}=$ $\left\{h^{*}(1), \ldots h^{*}(M)\right\}$ satisfies the following Bellman's equation [20]:

$$
\xi^{*}+h^{*}(i)=\max _{m \in \mathcal{M}}\left[\mathcal{R}(i, m)+\sum_{j=1}^{M} p_{i j} h^{*}(j)\right],
$$

where $h^{*}$ may have multiple solutions for this equation.

The optimal stationary policy $\mu^{*}$ can be found by using the policy-iteration algorithm. In the algorithm, we repeatedly execute policy-evaluation step and policy-improvement step until no further improvement is made [20].

\section{E. Suboptimal Heuristics}

Since solving the POMDP is computationally prohibitive, we adopt well-known heuristics based on the optimal policy of MDP. The simplest approach is to treat the most probable state (i.e., state with the highest belief value) as a true channel state. This is called the maximum-likelihood (ML) heuristic [21] and the corresponding suboptimal control policy can be stated as:

$$
\mu_{M L}^{*}=\mu_{M D P}\left(\arg \max _{i \in \mathcal{S}} \lambda_{i}(t)\right) .
$$

Although the ML heuristic is simple, it does not fully exploit the given belief state information. For example, the PHY mode chosen by the ML heuristic may not be optimal if we consider the entire belief state space. Therefore, as an alternative, we can use the voting heuristic [22] where each state elects the best PHY mode based on the optimal policy of MDP. Then, each voted PHY mode is weighted by the belief state value corresponding to the given state and PHY mode under consideration. The voting heuristic policy can be expressed as:

$$
\mu_{\text {voting }}^{*}=\arg \max _{m} \sum_{i \in \mathcal{S}} \lambda_{i}(t) \delta\left(\mu_{M D P}(i)-m\right) .
$$

\section{Performance Evaluation}

In this section, we evaluate the proposed scheme using MATLAB-based simulation. To demonstrate the effectiveness and utility, we compare the performance of our scheme with existing well-known rate adaptation schemes, under various fading conditions. We also study the performance impact of the number of FSMC states and average channel conditions.

\section{A. Simulation Setup}

We consider a simple communication scenario with a transmitter-receiver pair in IEEE 802.11a WLANs. We assume that the large-scale signal propagation effects (e.g., path loss, shadowing) are handled in an average sense, and Rayleigh fading dominates the time-varying channel condition. We consider the FSMC with various numbers of states (i.e., $M=5,7,10,15$ ); 


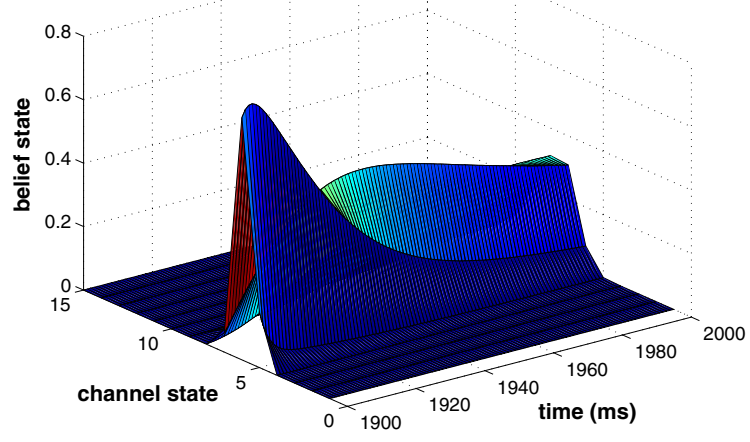

Fig. 7. Example dynamics of the belief state vector

and the SNR thresholds of the FSMC are determined so that all states have an identical steady-state probability.

Throughout the simulation, the data payload size $L$ is fixed, so the PPDU size is also fixed at PPDU_length $=900$ bytes. No data frame fragmentation is assumed in packet transmission. The transmitter supports the 8 PHY modes (see Section III). It transmits each packet with the same fixed power level. Since we use a slotted time model for the fading process in the POMDP formulation, we need to set the duration of each time slot close to the average frametransmission interval. We set slot_duration $=1 \mathrm{~ms}$ since the average transmission time for a 900-byte packet with PHY mode 1 (i.e., BPSK with rate- $1 / 2$ convolutional code) is 1.0215 ms. We assume that the frame errors due to collisions are always correctly differentiated from the errors due to channel conditions [23], [24]. ${ }^{4} \mathrm{We}$ also assume that $p_{a c k} \approx 0$, which is reasonable in practice, because of its small frame size and the associated PHY mode (i.e., BPSK), which is robust to error. Each simulation is run for 50 seconds.

To demonstrate the benefits of our scheme, we evaluate the performance of the following rate adaptation schemes: (i) the proposed scheme; (ii) ARF-3; (iii) ARF-10; (iv) adaptive ARF (AARF); and (v) an IDEAL scheme. The proposed scheme uses the voting heuristic described in Section IV. In ARF3(ARF-10), the transmitter increases the PHY rate after 3(10) consecutive successful transmissions, and it decreases the PHY rate after 2 consecutive transmission failures in both schemes. AARF is an enhanced version of ARF in which the transmitter adaptively changes the probing interval so as to work well in both slow- and fast-fading environments. In AARF, the transmitter immediately falls back to the previous PHY rate, and doubles the probing interval upon failure of the probing attempt. Like ARF, the transmitter sets the probing interval to the default value, 10, and decreases the PHY rate after observing two consecutive transmission failures. The probing interval upper bound is set to max_probing_interval $=50$. In the IDEAL scheme, we assume that the channel condition (i.e., SNR) is perfectly known to the transmitter. In this case,

\footnotetext{
${ }^{4}$ If there is no such differentiation, our scheme may suffer significantly since the prediction error in the belief states will propagate to the following states.
}

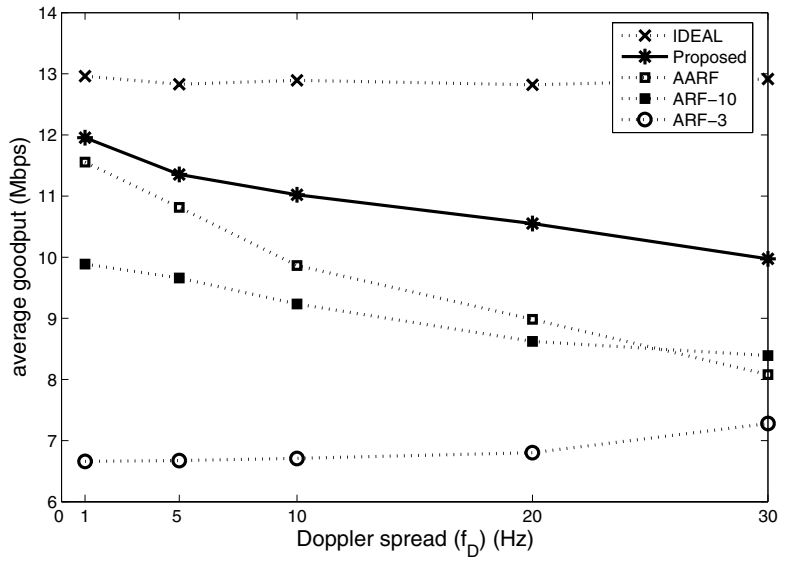

Fig. 8. Comparison of goodput among various rate adaptation schemes

the stationary policy of MDP (i.e., $\mu_{M D P}$ ) is used to direct the PHY mode for each transmission attempt. Therefore, the achieved goodput of the IDEAL scheme can be seen as a performance upper bound of the POMDP optimal policy. Therefore, this will be used as a reference in our simulation study. We use the average achieved goodput (in Mbps) as the main performance metric.

\section{B. Channel State Estimation Accuracy}

Before discussing the simulation results, we first show the accuracy of the belief state vector by giving an example as shown in Fig. 7, which illustrates the dynamics of the belief state in time period $[1900,2000]$ (ms) of our simulation. During this time period, the actual channel SNR monotonically (but not linearly) increases from $12.54 \mathrm{~dB}$ to $13.02 \mathrm{~dB}$, which belongs to the state 6 in the 15-state FSMC model, and there is no transmission failure during this time period. Fig. 7 shows that the highest value component of the belief vector migrates from state 6 to state 7 as the transmitter continuously observes successful frame transmissions, thus demonstrates the accuracy of the belief states. Later in this section, we will show how the belief state vector directs the selection of an optimal PHY mode, thus improving system performance.

\section{Effect of Fading Conditions}

Fig. 8 shows the average achieved goodput of the testing schemes under various fading conditions (i.e., Doppler spread values). As expected, the proposed POMDP-based rate adaptation scheme significantly outperforms the others for all simulated scenarios because of its ability to exploit the underlying channel fading characteristics. Our scheme achieves up to 92 $\%$ of the performance upper bound (i.e., the achieved goodput by the IDEAL scheme) with a small Doppler spread (i.e., slow-fading), and the performance degrades as the Doppler spread value increases (i.e., fast-fading). This is because the accuracy of the FSMC-based channel state prediction degrades rapidly as the channel fading process becomes faster, due to the increase in state transition probabilities. Note the FSMC model is not valid any more if the Doppler spread value is beyond a certain threshold (e.g., $\left.f_{D}>30(\mathrm{~Hz})\right)$.

The simulation results show that ARF-10 works well for slow-fading channels, however, the performance degrades as 


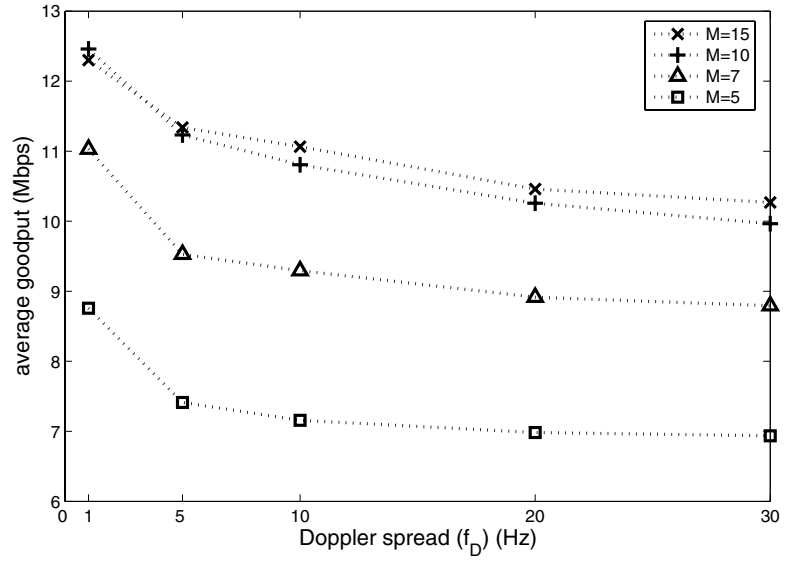

Fig. 9. Achieved goodput of the proposed scheme with various numbers of FSMC states under the normal channel condition

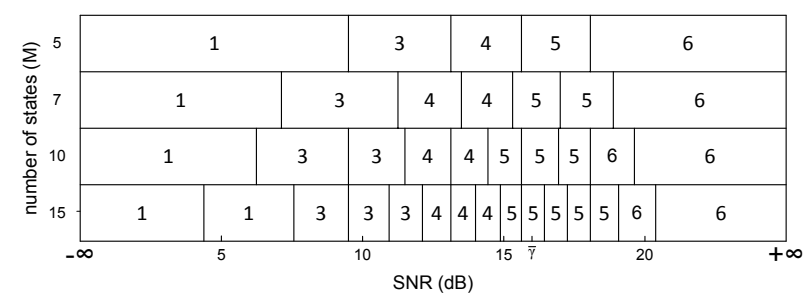

Fig. 10. The optimal PHY control policies for MDP with various numbers of FSMC states under the normal channel condition

the Doppler spread increases. This is because the transmitter cannot adapt quickly to fast-varying channel conditions due to the fixed probing interval of 10 . On the other hand, ARF3 performs poorly in case of slow-fading channels since it attempts rate increase too frequently, incurring a large number of transmission failures. It should be noted that ARF-10 outperforms ARF-3 since we simulated only slowly-varying fading channels. Similar to ARF-10, AARF shows good performance for slow-fading channels and the performance degrades as the Doppler spread increases. However, AARF performs slightly better than ARF-10, mainly because it is flexible in adjusting the probing interval to some extent.

\section{Effect of the Number of FSMC States}

We study the effect of the number of FSMC states by comparing the goodput of the proposed scheme under various FSMC models of different numbers of states. We consider four FSMC models with different numbers of states, i.e., $M=5,7,10,15$. In addition, we consider two average channel conditions, i.e., normal with $\bar{\gamma}=16$ (dB), and good with $\bar{\gamma}=22(\mathrm{~dB})$, to see how the FSMC model with different numbers of states performs under different average channel conditions. Note that, under a bad channel condition (e.g., $\bar{\gamma}<10(\mathrm{~dB}))$, there will be no substantial benefit of using a large number of states since in such a case, only low PHY rates (i.e., BPSK or QPSK) will be used for most of the time.

Fig. 9 shows the effect of the number of states on goodput performance with the corresponding optimal MDP policies as shown in Fig. 10, under the normal channel condition (i.e., $\bar{\gamma}=16(\mathrm{~dB}))$. As shown in Fig. 9, significant performance improvements result as the number of state increases from 5

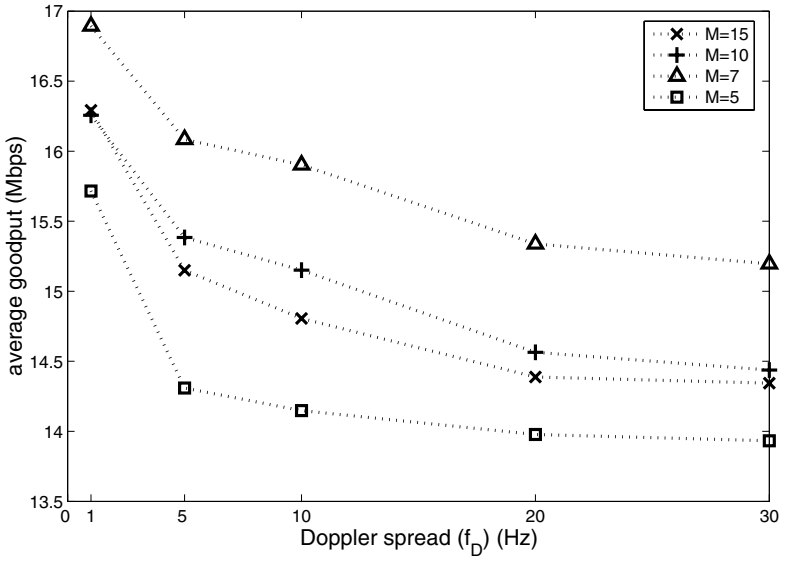

Fig. 11. Achieved goodput of the proposed scheme with various numbers of states under the good channel condition

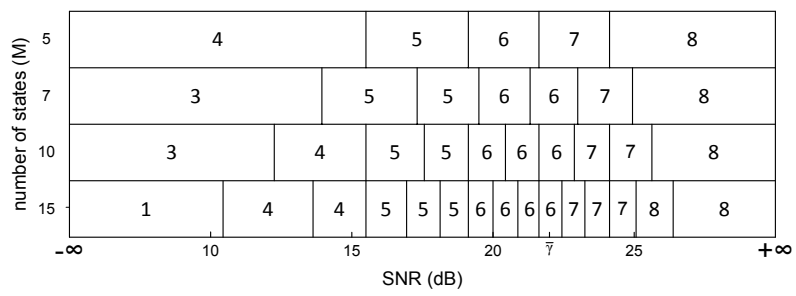

Fig. 12. The optimal PHY control policies for MDP with various numbers of FSMC states under the good channel condition

to 10 . This is because a large number of states provides finergrained SNR intervals, and thus enables better PHY mode selection decisions. However, there is virtually no performance improvement as the number of states increases further from 10 to 15 because there is no substantial difference in PHY mode decision policies as shown in Fig. 10. In other words, the impact of the number of states on performance would become negligible if it increases beyond a certain threshold.

Fig. 11 shows that there is no significant difference in goodput performance under the good channel condition (i.e., $\bar{\gamma}=22(\mathrm{~dB}))$. Interestingly, even the FSMC model with a smaller number of states (i.e., $M=7$ ) performs better than those with a larger number of states (i.e., $M=10,15$ ). This is because, under a good channel condition, the number of state does not have any significant impact on the goodput since the high PHY rates would be used for most of the time regardless of the number of states, as shown in Fig. 12. The SNR thresholds of FSMC, in fact, affects the quality of PHY mode control policy which is the main reason why the achieved goodput does not increase monotonically with model complexity (i.e., the number of states). The issue of finding the optimal number of states is part of our future study.

\section{E. Comparison of the PHY mode control}

Fig. 13 clearly demonstrates the effectiveness of the proposed scheme by comparing the PHY mode adaptation of the five rate adaptation schemes under consideration. Fig. 13(a) shows the observed channel SNR variation with Doppler spread $f_{D}=10(\mathrm{~Hz})$ during the time period [1500,1700] (ms) in the simulation. Figs. 13(b) and (c) show the behavior of 

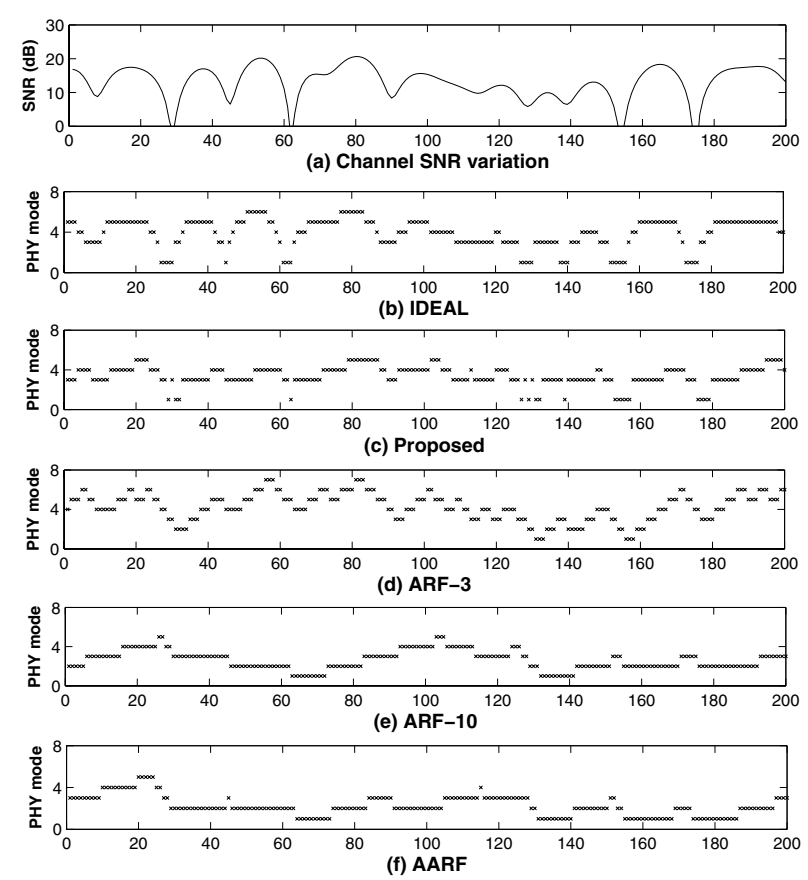

Fig. 13. Comparison of PHY mode adaptation of the schemes under consideration

IDEAL and proposed schemes, respectively. Since the IDEAL scheme operates using the optimal MDP policy based on completely observable channel states, it perfectly adapts the PHY mode to the fluctuating channel conditions. The proposed scheme also directs the PHY mode in close accordance with the channel condition. However, it is slow in up-shifting the PHY mode as one can observe, for instance, in time duration $[5,20](\mathrm{ms})$. This is because the transmission failure due to the degraded channel condition changes the belief state drastically, while consecutive transmission successes slightly change the belief state. Figs. 13(d),(e) and (f) show the behaviors of ARF3 , ARF-10 and AARF, respectively. ARF-3 is error-prone due mainly to its frequent probing attempts, while ARF-10 and AARF show lack of agility in rate adaptation.

\section{CONCLUSION AND FUtURE WORK}

In this paper, we proposed cross-layer transmission control that maximizes the expected goodput by exploiting the underlying channel fading characteristics. We assume channels to experience Rayleigh fading, which is modeled as a finitestate Markov channel (FSMC). We formulate the transmission control (i.e., PHY mode adaptation) problem as a POMDP where the PHY mode is selected based on the belief state, which is updated for each frame transmission using a simple Bayes rule. We compare the performance of the proposed scheme against four different rate-adaptation schemes: ARF3, ARF-10, AARF, and IDEAL. Our simulation results show that the proposed scheme outperforms the two well-known rate adaptation schemes (i.e., ARF and AARF) thanks to its ability to closely track the actual channel condition by exploiting the underlying channel fading process.

Our future work includes finding an efficient mechanism for Markov channel modeling, which is one of the main chal- lenges of the proposed scheme, and finding the optimal SNR thresholds of the FSMC model. It also includes investigating the PHY mode control strategies with various performance metrics (e.g., delay and power consumption).

\section{ACKNOWLEDGEMENT}

The work reported in this paper was supported in part by NSF grants CNS-0519498 and CNS-0721529, and an Intel Communications Research grant.

\section{REFERENCES}

[1] Wireless LAN Medium Access Control (MAC) and Physical Layer (PHY) Specifications, IEEE Std. 802.11, Aug 1999.

[2] G. Holland, N. Vaidya, and P. Bahl, "A Rate-Adaptive MAC Protocol for Multi-Hop Wireless Networks," in Proc. ACM MobiCom '01, 2001, pp. 236-251.

[3] B. Sadeghi, V. Kanodia, A. Sabharwal, and E. Knightly, "Opportunistic Media Access for Multirate Ad Hoc Networks," in Proc. ACM MobiCom '02, 2002, pp. 24-35.

[4] A. Kamerman and L. Monteban, "WaveLAN II: A High-Performance Wireless LAN for the Unlicensed Band," Bell Labs Technical Journal, pp. 118-133, Summer 1997.

[5] M. Lacage, M. H. Manshaei, and T. Turletti, "IEEE 802.11 Rate Adaptation: A Practical Approach," in Proc. ACM MSWiM '04, 2004, pp. 126-134.

[6] J. Bicket, "Bit-rate Selection in Wireless Networks," MIT Master's Thesis, 2005.

[7] S. H. Y. Wong, H. Yang, S. Lu, and V. Bharghavan, "Robust Rate Adaptation for 802.11 Wireless Networks," in Proc. ACM MobiCom '06, 2006, pp. 146-157.

[8] D. Aguayo, J. Bicket, S. Biswas, G. Judd, and R. Morris, "Link-level Measurements from an 802.11b Mesh Metwork," SIGCOMM Computer Communication Review, vol. 34, no. 4, pp. 121-132, 2004.

[9] A. Goldsmith, Wireless Communications. Cambridge University Press, 2005.

[10] H. S. Wang and N. Moayeri, "Finite-State Markov Channel - A Useful Model for Radio Communication Channels," IEEE Transactions on Vehicular Technology, vol. 44, no. 1, pp. 163-171, Feb 1995.

[11] H. S. Wang and P.-C. Chang, "On Verifying the First-Order Markovian Assumption for a Rayleigh Fading Channel Model," IEEE Transactions on Vehicular Technology, vol. 45, no. 2, pp. 353-357, May 1996.

[12] W. Turin and R. van Nobelen, "Hidden Markov Modeling of Flat Fading Channels," IEEE Journal on Selected Areas in Communications, vol. 16, no. 9, pp. 1809-1817, Dec 1998.

[13] Q. Liu, S. Zhou, and G. B. Giannakis, "Queuing With Adaptive Modulation and Coding Over Wireless Links: Cross-Layer Analysis and Design," IEEE Transactions on Wireless Communications, vol. 4, no. 3, pp. 1142-1153, May 2005.

[14] R. K. Guha and S. Sarkar, "Characterizing Temporal SNR Variation in 802.11 Networks," in Proc. IEEE WCNC '06, Apr 2006, pp. 1408-1413.

[15] J. A. Hartwell and A. O. Fapojuwo, "Modeling and Characterization of Frame Loss Process in IEEE 802.11 Wireless Local Area Networks," in Proc. IEEE VTC '04, Sep 2004, pp. 4481-4485.

[16] S. A. Khayam and H. Radha, "Markov-based Modeling of Wireless Local Area Networks,' in Proc. ACM MSWiM '03, 2003, pp. 100-107.

[17] Intersil, "HFA3861B: Direct Sequence Spread Spectrum Baseband Processor," Jan 2000.

[18] D. Qiao, S. Choi, and K. G. Shin, "Goodput Analysis and Link Adaptation for IEEE 802.11a Wireless LANs," IEEE Transactions on Mobile Computing, vol. 1, no. 4, pp. 278-292, Oct-Dec 2002.

[19] O. Madani, S. Hanks, and A. Condon, "On the Undecidability of Probabilistic Planning and Related Stochastic Optimization Problems," Artificial Intelligence, vol. 147, no. 1-2, pp. 5-34, 2003.

[20] D. P. Bertsekas, Dynamic Programming and Optimal Control: Vol I and II. Athena Scientific, 2005.

[21] I. Nourbakhsh, R. Powers, and S. Birchfield, "DERVISH: An OfficeNavigating Robot," AI Magazine, vol. 16, no. 2, 1995.

[22] R. Simmons and S. Koenig, "Probabilistic Robot Navigation in Partially Observable Environments,' in Proc. IJCAI '95, July 1995, pp. 10801087.

[23] Q. Pang, V. C. Leung, and S. C. Liew, "A Rate Adaptation Algorithm for IEEE 802.11 WLANs Based on MAC-Layer Loss Differentiation," in IEEE WCNC '05, Oct 2005, pp. 709-717.

[24] X. Chen, D. Qiao, J. Yu, and S. Choi, "Probabilistic-Based Rate Adaptation for IEEE 802.11 WLANs," in IEEE GLOBECOM '07, Nov 2007, pp. 4904-4908. 